\title{
Extending Maple Capabilities for Solving and Displaying Inequalities
}

\author{
A. Iglesias ${ }^{1, \star}$ and R. Ipanaqué ${ }^{2}$ \\ ${ }^{1}$ Department of Applied Mathematics and Computational Sciences, \\ University of Cantabria, Avda. de los Castros, \\ s/n, E-39005, Santander, Spain \\ iglesias@unican.es \\ http://personales.unican.es/iglesias \\ 2 Department of Mathematics, National University of Piura, \\ Urb. Miraflores s/n, Castilla, Piura, Perú \\ robertipanaquechero@yahoo.es \\ http://www . unp.edu.pe/pers/ripanaque
}

\begin{abstract}
Solving inequalities is a very important topic in computational algebra. This paper presents a new Maple package, IneqGraphics, for displaying the two-dimensional solution sets of several inequalities of real variables. The package also deals with inequalities involving complex variables by displaying the corresponding solutions on the complex plane. The package provides graphical solutions to many inequalities (such as those involving polynomial, rational, logarithmic, exponential and trigonometric functions) that cannot be solved by using the standard Maple commands, which are basically reduced to linear inequalities. In addition, our outputs are consistent with Maple's notation and results in the sense that the package provides a similar output for those cases that can also be solved with Maple. To show the performance of the package, several illustrative and interesting examples are described.
\end{abstract}

\section{Introduction}

Solving inequalities is a very important topic in Mathematics, with outstanding applications in many problems of theoretical and applied science [1, 2, 4, 6,, 7 . However, since there is not a general methodolody for solving inequalities, their symbolic computation is still a challenging problem in computational algebra [3]. For example, Maple includes some commands for dealing with inequalities, but they are drastically limited to the linear case [5]. This drawback motivated us to create a Maple package, IneqGraphics, that is introduced in this paper. The package displays the two-dimensional solution sets of several inequalities. Typically, in our package the input might be comprised of any sequence of inequalities (connected by either the and or or operators) involving nonlinear expressions such as polynomial, rational, trigonometric, exponential and logarithmic functions - that cannot be solved by using the standard Maple commands. The

\footnotetext{
^ Corresponding author.
} 
package also deals with inequalities involving complex variables by displaying the corresponding solutions on the complex plane.

The structure of this paper is as follows: Section 2 describes the main standard Maple tools for solving inequalities. Then, Section 3 introduces the new Maple package, IneqGraphics, and describes the commands implemented within. The performance of the package is also discussed in this section by using some illustrative examples. Finally, Section 4 closes with the main conclusions of this paper and some further remarks.

\section{Standard Maple Tools for Solving Inequalities}

Maple incorporates only a few commands for solving inequalities. The command solve (ineq,var), solves one inequality in one variable, var, for all the cases where the equality points of ineq can be properly ordered. While it is not required for the inequality to be linear (for instance, you can solve $e^{x}>x+1$ ) it only accepts one inequality, thus strongly limiting its applicability.

In addition, the Maple 10 package plots provides the command inequal that plots regions defined by logical combinations of linear inequalities in the $\mathbb{R}^{2}$ space. Although its input allows us to enter more than one equality or inequality, they must necessarily be linear. For example, the package fails to display the solution sets of each of the inequalities $x^{2}+y<1$ and $x^{2}+y^{2} \leq 16$ on the set $[-5,5] \times[-5,5]$ :

$>$ with (plots):

Warning, the name changecoords has been redefined

$>$ inequal $\left(\mathrm{x}^{\wedge} 2+\mathrm{y}<1, \mathrm{x}=-5 . .5, \mathrm{y}=-5 . .5\right)$;

Error, (in inequal) the inequalities should be linear in ' $x$ ' and ' $y$ '

$>$ inequal ( $\left.\mathrm{x}^{\wedge} 2+\mathrm{y}^{\wedge} 2<16, \mathrm{x}=-5 . .5, \mathrm{y}=-5 . .5\right)$;

Error, (in inequal) the inequalities should be linear in ' $x$ ' and ' $y$ '

This problem has been partially overcome in [8] where the author presents an interesting extension of the command inequal to the case of nonlinear expressions. In particular, his new command inequalities admits systems of nonlinear inequalities, including the case of expressions given in polar coordinates. However, the package does not solve the problem in all its generality as the inequalities can only be connected by the and operator, not all nonlinear expressions can be considered and the polar form fails in presence of loops, multivalued curves and self-intersection scenarios, to mention some of the limitations we noticed.

The package IneqGraphics, described in the next section, overcomes many of those limitations and allows the user to solve a large family of real and complex inequality systems and equations and display their two-dimensional solution sets accordingly. 


\section{The Package IneqGraphics: Some Illustrative Examples}

As shown in the previous section, inequalities involving nonlinear functions cannot be visualized by means of the inequal command. They can be solved, however, by loading the package developed by the authors:

> libname:="C: \\Program files $\backslash \backslash$ Maple 10/mypackages", libname:

$>$ with(IneqGraphics);

[complexineqplot, ineqplot]

which includes the command

$$
\text { ineqplot (ineqs, } x=x m i n . x \operatorname{xax}, y=y m i n . y m a x \text {, ops) }
$$

for displaying the two-dimensional region of the set of points satisfying the inequalities ineqs of real numbers inside the square [xmin, xmax] $\times[y m i n$, ymax]. For example, the inequalities indicated in the previous section can be solved as follows:

$>$ ineqplot $\left(x^{\wedge} 2+y<1, x=-5 . .5, y=-5 . .5\right)$;

See Figure 1(left)

$>$ ineqplot $\left(\mathrm{x}^{\wedge} 2+\mathrm{y}^{\wedge} 2<=16, \mathrm{x}=-5 . .5, \mathrm{y}=-5 . .5\right)$;

See Figure 11(right)
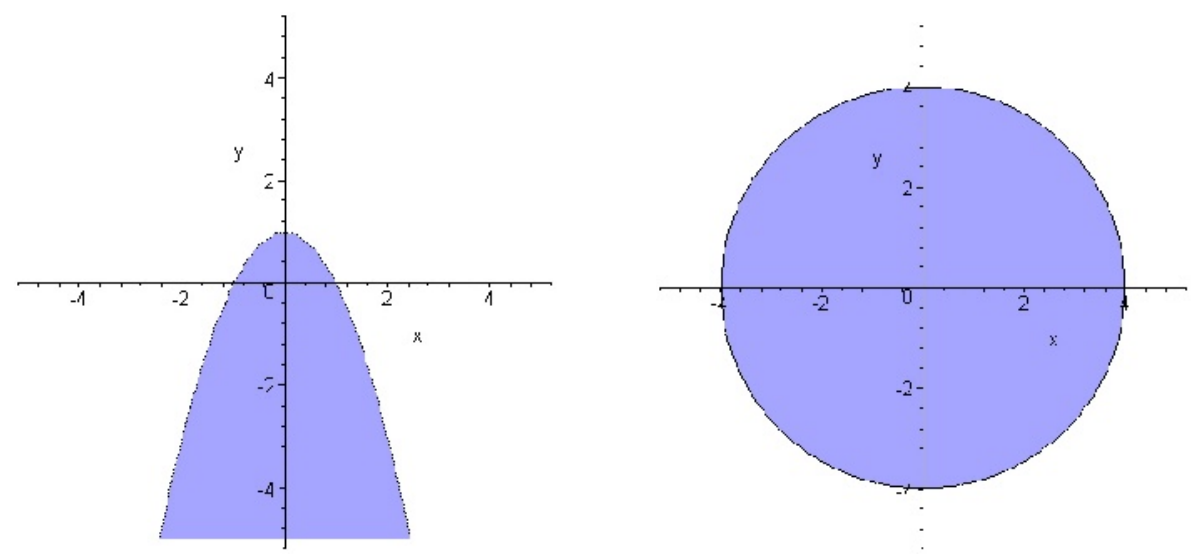

Fig. 1. Some examples of inequality solutions on the square $[-5,5] \times[-5,5]$ : (left) $x^{2}+y<1 ;$ (right) $x^{2}+y^{2} \leq 16$

Similarly, Fig. 2 displays the solution sets of the inequalities $1<x^{2}+y^{2}<4$ and $\sin \left(x^{2}+y^{2}\right) \geq \frac{1}{2}$ on the squares $[-2.5,2.5] \times[-2.5,2.5]$ and $[-4,4] \times[-4,4]$, respectively. Note that the Maple command inequal does not provide any solution for these inequalities either. 
$>$ ineqplot $\left(\left\{1<x^{\wedge} 2+y^{\wedge} 2, x^{\wedge} 2+y^{\wedge} 2<4\right\}, x=-2.5 \ldots 2.5, y=-2.5 \ldots 2.5\right)$;

See Figure Q (left)

>ineqplot $\left(\sin \left(x^{\wedge} 2+y^{\wedge} 2\right)>=1 / 2, x=-4 \ldots 4, y=-4 \ldots 4\right.$, feasiblepoints $=50$, linespoint $s=2500$ );

See Figure 2(right)
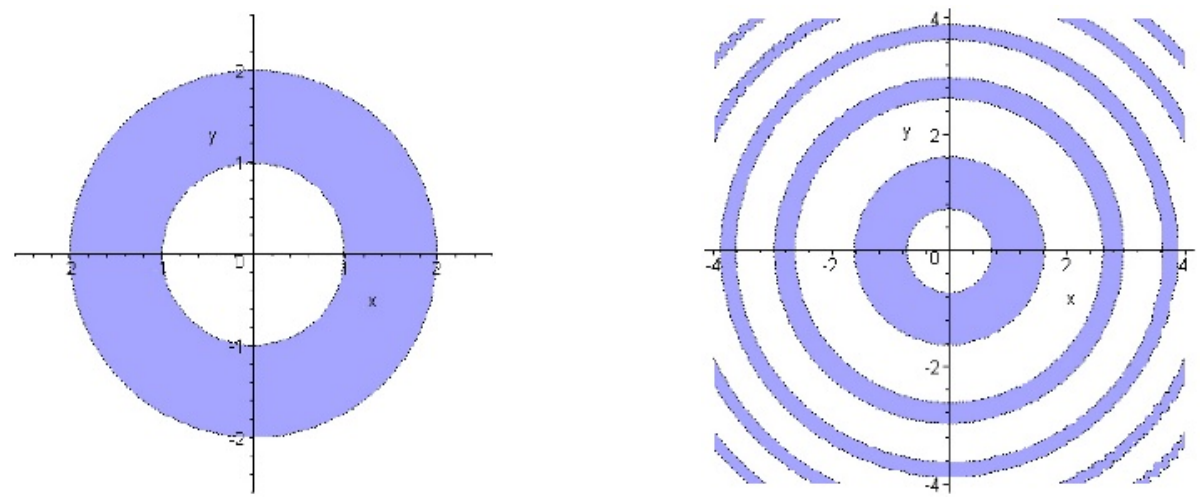

Fig. 2. Some examples of inequality solutions: (left) $1<x^{2}+y^{2}<4$ on the square $[-2.5,2.5] \times[-2.5,2.5] ;$ (right) $\sin \left(x^{2}+y^{2}\right) \geq \frac{1}{2}$ on the square $[-4,4] \times[-4,4]$

As mentioned above, our input can be comprised of several inequalities combined by using the and and or operators simultaneously. The next two examples are aimed to show this fact:

$>\operatorname{ineqplot}(1<\operatorname{abs}(\mathrm{x})+\mathrm{abs}(\mathrm{y})$ and $\operatorname{abs}(\mathrm{x})+\mathrm{abs}(\mathrm{y})<=2$ or $\operatorname{abs}(\mathrm{x}+\mathrm{y})<1 / 2$, $\mathrm{x}=-3 . .3, \mathrm{y}=-3 . .3$, linespoint $\mathrm{s}=400$, feasiblepoint $\mathrm{s}=35$ );

See Figure 3 (left)

$>$ ineqplot $\left((\mathrm{x}+2)^{\wedge} 2+\mathrm{y}^{\wedge} 2<=1\right.$ or $(\mathrm{x}-2)^{\wedge} 2+\mathrm{y}^{\wedge} 2<=1$ or $\left((\mathrm{x}+2)^{\wedge} 2+\mathrm{y}^{\wedge} 2>=3\right.$ and $\left.(\mathrm{x}-2)^{\wedge} 2+\mathrm{y}^{\wedge} 2>=3\right), \mathrm{x}=-4 . .4, \mathrm{y}=-2 . .2$, feasiblepoint $\left.\mathrm{s}=30\right)$;

See Figure 3 (right)

The previous command, ineqplot, can be generalized to inequalities involving complex numbers. The new command:

complexineqplot (ineqs, $z=($ Rezmin . Rezmax, Imzmin. . Imzmax), opts)

displays the solution sets of the inequalities ineqs of complex numbers inside the square in the complex plane given by [Rezmin, Rezmax] $\times$ [Imzmin, Imzmax]. In this case, the functions appearing within the inequalities need to be real-valued functions of a complex argument, e.g. Abs, Re and $I m$. For example:

$>$ complexineqplot $\left(\left\{1<\operatorname{abs}\left(z^{\wedge} 2-z+1\right), \operatorname{abs}\left(z^{\wedge} 2-z+1\right)<4\right\}\right.$, $z=(-2 \ldots 3 .,-3 \ldots 3$.$) , feasiblepoints =25)$; 

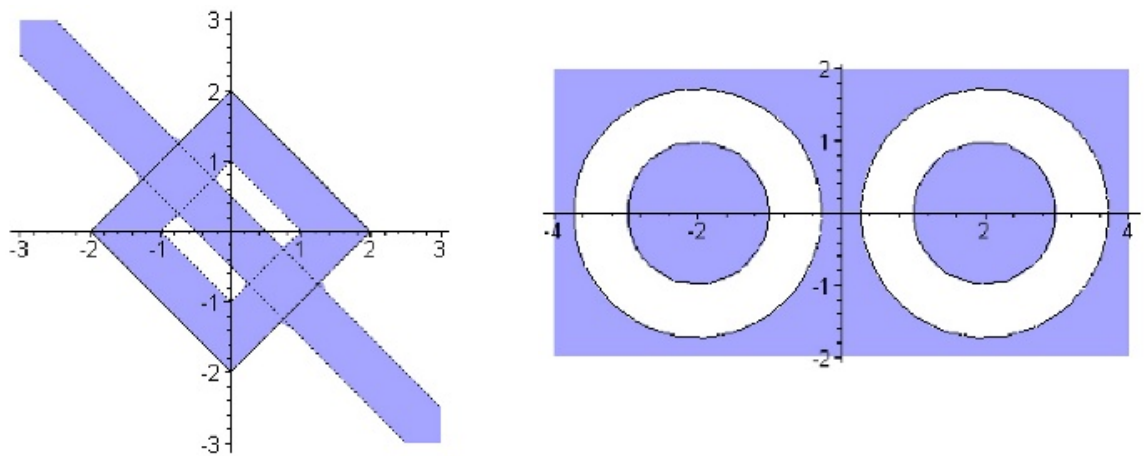

Fig. 3. Some examples of inequality solutions connected with the and and or operators: (left) $1<|x|+|y| \leq 2$ or $|x+y|<\frac{1}{2}$ on the square $[-3,3] \times[-3,3]$; (right) $(x+2)^{2}+y^{2} \leq$ 1 or $(x-2)^{2}+y^{2} \leq 1$ or $(x+2)^{2}+y^{2} \geq 3$ or $(x-2)^{2}+y^{2} \geq 3$ on the square $[-4,4] \times[-2,2]$

\section{See Figure 4 (left)}

$>$ complexineqplot $(\{1<\operatorname{abs}(\sin (z-2)+z), \operatorname{abs}(\sin (z-2)+z)<4\}$, $z=(-5 \ldots 8 .,-4 \ldots 4$.$) , feasiblepoints =35$, linespoints $=550)$;

\section{See Figure 4 (right)}

We would like to point out that the output obtained from the package is consistent with Maple's notation and results in the sense that the package provides a similar output for those cases already solved by Maple. Figure 5 illustrates this fact: on the left, the solutions provided by the standard Maple command
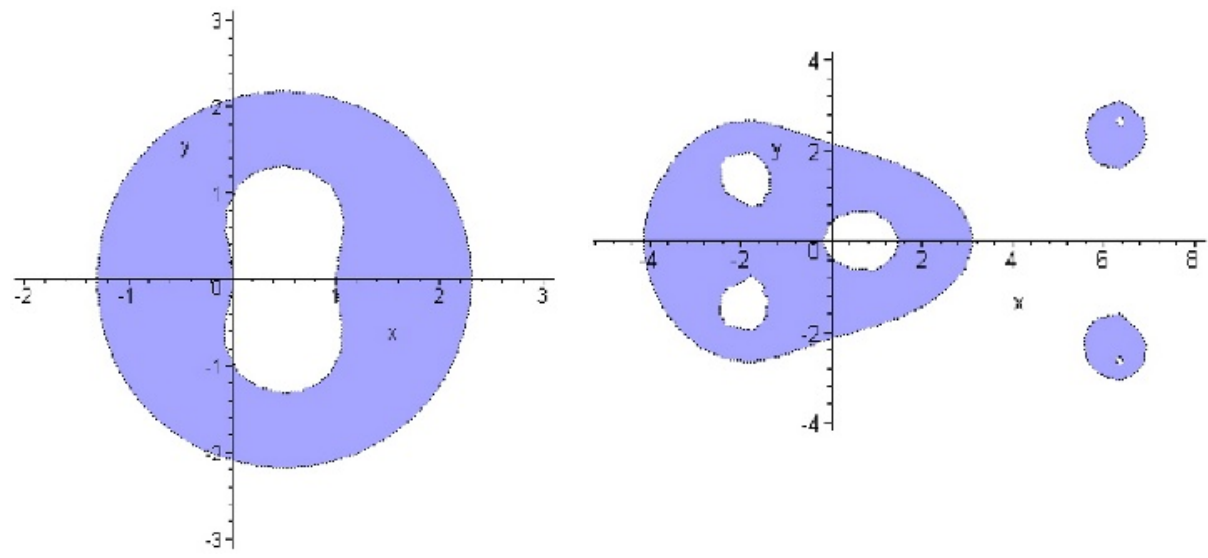

Fig. 4. Some examples of inequality solutions for $z \in \mathbb{C}$ : (left) $1<\left\|z^{2}-z+1\right\|<4$, $\operatorname{Re}(z) \in[-2,3], \operatorname{Im}(z) \in[-3,3] ;$ (right) $1<\|\sin (z-2)+z\|<4, \operatorname{Re}(z) \in[-5,8]$, $\operatorname{Im}(z) \in[-4,4]$ 

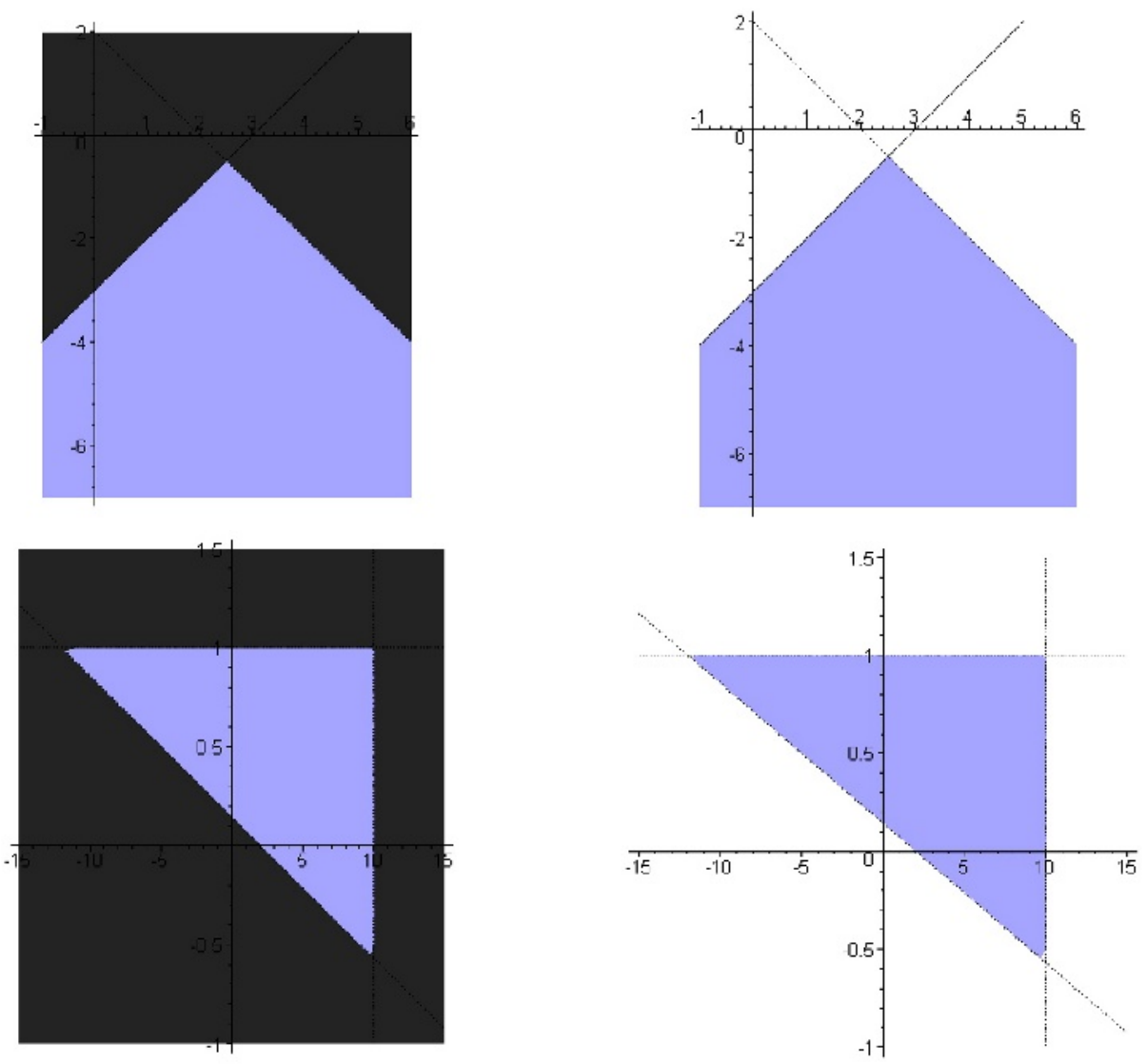

Fig. 5. Solution sets for the inequality systems: (top) $x+y \leq 2$ and $x-y \geq 3$; (bottom) $\frac{x}{2}+7 y>1$ and $y<1$ and $x<10$. The solutions have been obtained by applying: (left) the standard Maple command inequal; (right) the new command ineqplot.

inequal to the inequalities $x+y \leq 2$ and $x-y \geq 3$ (top) and $\frac{x}{2}+7 y>1$ and $y<1$ and $x<10$ (bottom) on $[-1,6] \times[-7,2]$ and $[-15,15] \times[-1,1.5]$ respectively are displayed. On the right, the solutions obtained by using the new command ineqplot are shown. As the reader can see, the new solution sets match perfectly those obtained from the standard Maple commands.

The last examples aim to show how complicated the inequality systems can be: in addition to include polynomial, rational, exponential, logarithmic and trigonometric functions, combinations and even compositions of these (and other) functions can also be considered. In Figures 6 and 7 the solutions sets of the inequality systems:

$$
\begin{gathered}
x^{2}+y^{2}<5 \text { and }\left|\log \left(x^{2}+1\right)+y\right|>1 \\
x^{2}-y<2 \text { and } e^{x}+e^{y}>1 \text { and } e^{x}+e^{y}<4
\end{gathered}
$$



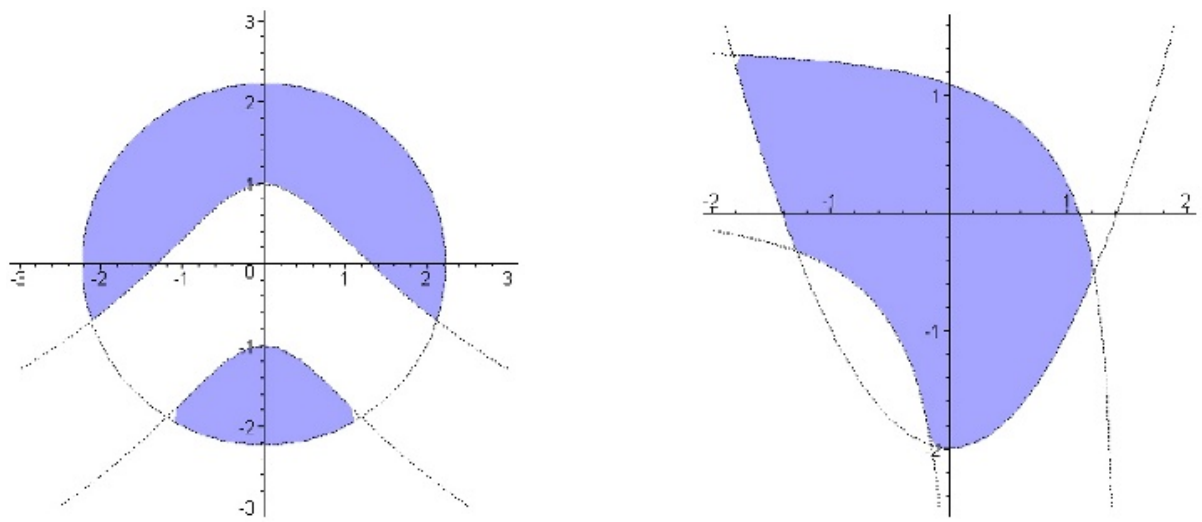

Fig. 6. (left, right) Solution sets for the inequality systems given by (1) and (2) respectively
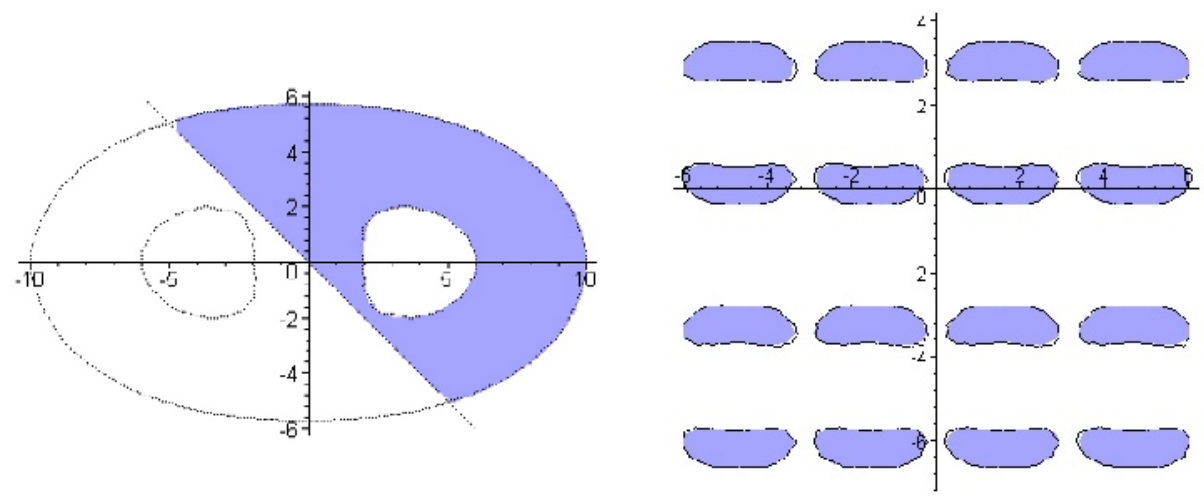

Fig. 7. (left, right) Solution sets for the inequality systems given by (3) and (4) respectively

$$
\begin{gathered}
4-\left(\sqrt{\left(\sqrt{x^{2}+y^{2}}-4\right)^{2}+y^{2}}-4\right)^{2}>0 \text { and } x+y>0 \\
\sqrt{\sin ^{2}(x)+(\sin (y)-2)^{2}} \sqrt{(\sin (x)+1)^{2}+\sin ^{2}(y)} \sqrt{(\sin (x)-1)^{2}+\sin ^{2}(y)} \leq \frac{1}{8}
\end{gathered}
$$

on $[-3,3] \times[-3,3],[-2,2] \times[-2.5,1.6],[-10,10] \times[-6,6]$ and $[-6,6] \times[-7,4]$ respectively, are displayed.

\section{Conclusions and Further Remarks}

In this paper a new Maple package, IneqGraphics, to solve real and complex inequalities and display their associated two-dimensional solution sets is 
introduced. The package extends Maple's capabilities by providing graphical solutions to many inequalities (such as those involving polynomial, rational, trigonometric, exponential and logarithmic functions) that cannot be solved by using the standard Maple commands and packages. The performance of the package has been illustrated by means of several interesting examples. In all the cases, the output obtained is consistent with Maple's notation and results in the sense that the package provides a similar output for those cases already solved by Maple. We are currently working on the extension of these results to the three-dimensional case and the implementation of new routines for other kind of inequalities by using quantifier elimination (QE) and cylindrical algebraic decomposition (CAD) algorithms. The obtained results will be reported elsewhere.

\section{References}

1. Beckenbach, E.F., Bellman, R.E.: An Introduction to Inequalities. Random House, New York (1961)

2. Hardy, G.H., Littlewood, J.E., Pólya, G.: Inequalities (Second Edition). Cambridge University Press, Cambridge (1952)

3. Ipanaque, R., Iglesias, A.: A Mathematica Package for Solving and Displaying Inequalities. Lecture Notes in Computer Science, 3039 (2003) 303-310

4. Kazarinoff, N.D.: Geometric Inequalities. Random House, New York (1961)

5. Maple User Manual. Maple Waterloo Inc., Canada (2005)

6. Mitrinovic, D.S.: Analytic Inequalities. Springer-Verlag, New York (1970)

7. Mitrinovic, D.S., Pecaric, J.E., Volenec, V.: Recent Advances in Geometric Inequalities. Kluwer, Dordrecht (1989)

8. Web page: http://www.math.ubc.ca/ ${ }^{\sim}$ israel/advisor/advisor6/h9r1.htm 